\title{
A Novel Electrochemical Enzyme Biosensor for Detection of 17 $\beta$ - Estradiol by Mediated Electron-transfer System.
}

\author{
Anqing Wang ${ }^{1}$, Yaping Ding ${ }^{1}$, Li Li $i^{1}$, Yuxin He ${ }^{1}$, Chaolin Wang ${ }^{1}$, Qianwen Mei', Dingding Duan ${ }^{1}$ \\ ${ }^{1}$ Department of Chemistry, Shanghai University, Shangda Road, Baoshan District, Shanghai 200444, P.R.C \\ Corresponding e-mail address: wdingyp@sina.com, lilidu@shu.edu.cn
}

\begin{abstract}
:
In this work, an extremely sensitive enzyme sensor for detection of $17 \beta$-estradiol based on electropolymerized Llysine molecules on a glassy carbon electrode (GCE) modified with critic acid@graphene (CA-GR) and cross-linked with laccase enzyme has been developed in this work. The morphology of the enzyme modified electrode was characterized by TEM, SEM and FTIR. The electrochemical detection of $17 \beta$-Estradiol was successfully conducted by mediated electron-transfer system and detection limit of $17 \beta$-Estradiol was as low as $0.13 \mathrm{pM}$. The human urine samples analysis confirmed the application of this enzyme sensor to quantitative analysis of ultra-trace hormone.
\end{abstract}

Keywords: laccase enzyme, poly L-lysine, 17ß-Estradiol, thionine, electrochemical sensor.

\section{Introduction}

$17 \beta$-Estradiol (E2), a natural steroid with estrogenic activity, excessive E2 remained in animal food like meat and milk will cause women's fertility problem, increase the risk of exposure to ovarian and breast cancer [1]. Therefore, studying $17 \beta$-Estradiol is significant for clinical analysis/diagnosis. Conventionally, various of methods, such as HPLC, immunological methods, gas chromatography, and chemiluminescence, have been developed for detecting 17ß-Estradiol [2]. However, these methods require complex precipitation procedures and it suffer from low sensitivity, time consumption and high cost [3]. Hence, it is extremely urgent to develop a simple, rapid and sensitive method for the determination of E2. Enzyme based on electrochemical sensor is one of the most promising sensors for ultra-trace detection in complex environment, due to its high specificity and excellent accurate.
In this work, a novel $17 \beta$-Estradiol sensor was fabricated by laccase loading with CA-GR and electro-polymerized L-lysine film modified glassy carbon electrode (Lac/PLLY/CA-GR/GCE) as sensing platform. The asprepared sensor showed good stability and low detection limit $(0.13 p M)$ for the determination of $17 \beta$-Estradiol.

\section{Characterization of Lac/Poly L-lysine/CA-GR/GCE}

Fig. 1 is the morphology and microstructure of different modified materials investigated by SEM and TEM. Fig.1A shows that the highly polydisperse sphere-like laccase molecules of $150 \mathrm{~nm} \pm 50 \mathrm{~nm}$ were coated on nanocomposite materials. Fig. $1 \mathrm{~B}$, the CA-GR film presents the flake-like and crimple shapes structure. Fig.1(C), poly L-lysine nanoparticles loads on crimple shape of CA-GR. After cross-linking laccase, the surface 
of GCE was covered with uniform laccase sphere which had been illustrated in Fig.1(D).

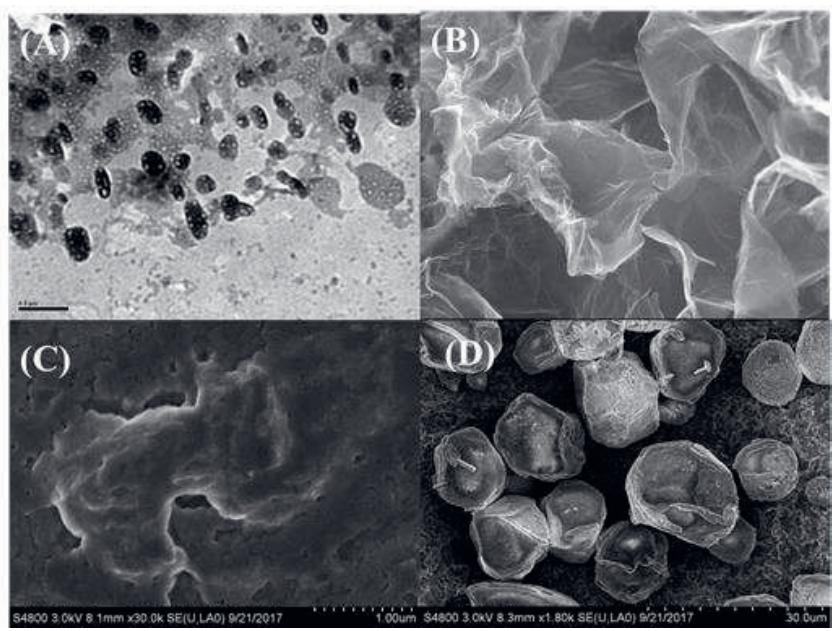

Fig.1. TEM images of (A) Lac/Plly/CA-GR/GCE and SEM images of (B) CA-GR/GCE, (C) Plly/CA-GR/GCE and (D) Lac/PIly/CA-GR/GCE

Fig. 2 (A) shows differential pulse voltammograms toward $0.5 \mathrm{pM} 17 \beta$-estradiol at a series of modified electrods, the For Lac/PIly/CA-GR/GCE, the peak current became larger than bare GCE obviously. Fig. 2(B) shows the electrochemical impedance spectroscopy of different modified electrodes. the CA-GR/GCE shows a small semicircular, which represents faster electron-transfer kinetics of [ $\mathrm{Fe}(\mathrm{CN}) 6$ ]3- / 4- compared to bare electrode. However, after the poly L-lysine films produced on the modified electrode surface, the Rct $(80.42 \Omega)$ was enlarged. When the laccase anchored on the Poly Llysine films, the Rct (1064.07 $\Omega$ ) was obviously enlarged, that because of its high selectivity electrochemical catalysis, what's more, its large size impede electron transferring.

\section{Experimental result}

As the Fig. 2(C) showed, differential pulse voltammograms toward $0.5 \mathrm{pM} 17 \beta$-estradiol in $0.1 \mathrm{M}$ sodium phosphate buffer, $\mathrm{pH} 7.0$ containing $1.2 \mathrm{mM}$ thionine as electrochemical mediator at bare GCE, CAGR/GCE, Poly L-ly/CA-GR/GCE, Poly L-ly/CAGR/Lac/GCE. For Lac/Poly L-ly/CA-GR/GCE, the peak current became larger than others obviously. Fig. 2B shows the amperometric signal of $17 \beta$-estradiol at different concentration ranging from $0.4 \mathrm{pM}$ to $57 \mathrm{pM}$ on Lac/Poly L-Iy/CA-GR/GCE. As shown in Fig. 2D, a linear regression equations: Ipa $=0.2364+0.197 \mathrm{c}(\mathrm{pM})$ $(R 2=0.992)$ was obtained. The detected limit was $0.13 p M$.
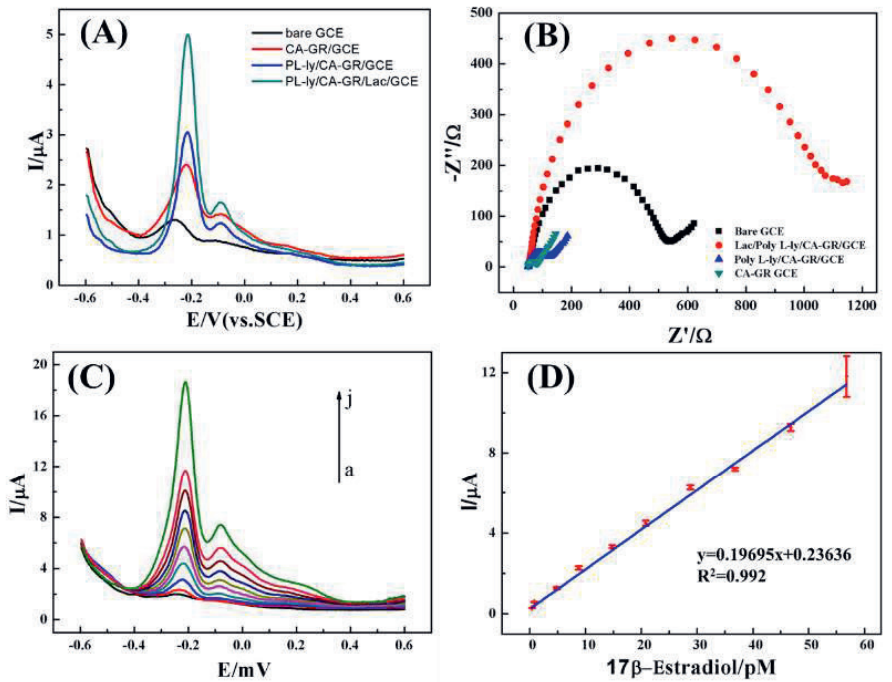

Fig. 2 (A) Electrochemical impedance spectra of different modified electrodes (B) DPV of 5pM $17 \beta$-estradiol in 0.1M PBS (pH 7.5) at bare GCE, CA-GR/GCE, Poly L-ly/CA-GR/GCE, Lac/Poly L-Iy/CA-GR/GCE. (C) DPV recorded at the enzyme sensor for $17 \beta$-estradiol concentrations form bottom to top $0.4 p M$ to $57 p M$. (D) Calibration curve constructed for $17 \beta$ estradiol at the Lac/Poly L-Iy/CA-GE/GCE.

Table. 1. Results of real sample analysis of Lac/PLLY/CAGR/GCE

\begin{tabular}{|c|c|c|c|c|}
\hline Samples & $\begin{array}{c}\text { Detected } \\
(\mu \mathrm{M})\end{array}$ & $\begin{array}{c}\text { Added } \\
(\mu \mathrm{M})\end{array}$ & $\begin{array}{c}\text { Found } \\
(\mu \mathrm{M})\end{array}$ & Recovery \\
\hline $\begin{array}{c}\text { Human } \\
\text { urine }\end{array}$ & - & 14.5 & 14.9 & $102.8 \%$ \\
\hline
\end{tabular}

This work is supported by the National Natural Science Foundation of China (No. 21671132)

\section{Reference}

[1] Y. Jia, Y. Peng, J. Bai, X. Zhang, Y. Cui, B. Ning, J. Cui, Z. Gao, Magnetic nanoparticle enhanced surface plasmon resonance sensor for estradiol analysis. Sensors and Actuators B: Chemical 254, 629-635 (2018); doi: 10.1016/j.snb. 2017.07 .061

[2] R. Gao, X. Cui, Y. Hao, L. Zhang, Y. Tang, A highlyefficient imprinted magnetic nanoparticle for selective separation and detection of $17 \mathrm{~b}$-estradiol in milk. Food Chemistry 194,1040-1047 (2016); doi: 10.1016 /j. foodch em.2015.08.112

[3] E. Povedano, F. Cincotto, C. Parrado, Decoration of reduced graphene oxide with rhodium nanoparticles for the design of a sensitive electrochemical enzyme biosensor for $17 \quad \beta$-estradiol. Biosensors and Bioelectronics 89, 343-351 (2017); doi: 10.1016/j.bios. 2016.07.018 